\title{
The road from systems biology to systems medicine
}

\author{
Olaf Wolkenhauer ${ }^{1,2}$, Charles Auffray ${ }^{3}$, Robert Jaster ${ }^{4}$, Gustav Steinhoff ${ }^{5}$ and Olaf Dammann ${ }^{6,7}$
}

\begin{abstract}
As research institutions prepare roadmaps for "systems medicine," we ask how this differs from applications of systems biology approaches in medicine and what we (should) have learned from about one decade of funding in systems biology. After surveying the area, we conclude that systems medicine is the logical next step and necessary extension of systems biology, and we focus on clinically relevant applications. We specifically discuss three related notions. First, more interdisciplinary collaborations are needed to face the challenges of integrating basic research and clinical practice: integration, analysis, and interpretation of clinical and nonclinical data for diagnosis, prognosis, and therapy require advanced statistical, computational, and mathematical tools. Second, strategies are required to (i) develop and maintain computational platforms for the integration of clinical and nonclinical data, (ii) further develop technologies for quantitative and time-resolved tracking of changes in gene expression, cell signaling, and metabolism in relation to environmental and lifestyle influences, and (iii) develop methodologies for mathematical and statistical analyses of integrated data sets and multilevel models. Third, interdisciplinary collaborations represent a major challenge and are difficult to implement. For an efficient and successful initiation of interdisciplinary systems medicine programs, we argue that epistemological, ontological, and sociological aspects require attention.
\end{abstract}

\section{LESSONS FROM SYSTEMS BIOLOGY}

Here, we discuss the developments that can or should take us from systems biology to systems medicine. Although it is easy to agree that medicine should embark on that journey, it remains unclear which route should be taken. Our own experience and analysis of developments in the field of systems biology has taught us several lessons of which the following are of central importance and which shall be the focus of our discussion: (i) many diseases have their origin in cellular malfunction, requiring a deep understanding of the mechanisms underlying cell functions; (ii) the emergence of diseases is a nonlinear dynamical phenomenon, requiring quantitative time-resolved monitoring of key biological parameters at the molecular, cellular, and physiological levels; (iii) advances in measurement technologies can generate large-scale, multilevel but also heterogeneous datasets, requiring not only new computational platforms to manage data but most importantly, requiring new ways of thinking, including the application and development of methodologies from the mathematical sciences; and (iv) to address clinical questions with statistical, mathematical, computational, molecular, and cell-biological methodologies requires strategic efforts to motivate and sustain cross-disciplinary collaborations.

Applying systems approaches in a clinical setting, practical, i.e., formal/legal and computational issues of data collection and sharing are the most immediate challenge and potential threat to progress. Although these issues are crucial and most pressing, we shall here emphasize the role of mathematical modeling as one aspect that is easily forgotten when it comes to setting priorities. Although the practical value of mathematical models is limited to very specific problems, and then comes with specific requirements for data collection, it should be noted that nonlinear dynamical phenomena can be understood only with the help of mathematical modeling as common sense and intuition will inevitably fail this aspect of biological complexity.

Finally, we urge a rethinking of systems biology as it develops toward systems medicine. Systems biology, which has emerged from a successful alliance of molecular and cell biology with computational and mathematical approaches, has been firmly set in a reductionist framework. Reductive approaches have allowed us to "zoom in" on molecular mechanisms underlying cell functions, accompanied by an ever-increasing degree of specialization. The flip side of this endeavor is fragmentation, data/evidence being tightly linked to a vast array of highly specialized technologies and a strong contextualization that limits conclusions to specific experimental/biological systems. We need to balance the pathway-centric approach, focusing on cellular mechanisms, by "zooming out" to actively seek law-like principles (e.g., of tissue organization). We believe that "integration" is a key concept that can serve as a basis for strategic developments that motivate a rethinking and the implementation of systems approaches in medicine.

Systems biology has been firmly established as an interdisciplinary approach, combining experimental work with mathematical modeling and computational analyses. The emergence of systems biology signaled a shift of focus from the identification of cellular components and their molecular characterization

\footnotetext{
'Department of Systems Biology and Bioinformatics, University of Rostock, Rostock, Germany; ${ }^{2}$ Stellenbosch Institute for Advanced Study (STIAS), Stellenbosch, South Africa; ${ }^{3}$ European Institute for Systems Biology \& Medicine, CNRS-UCBL-ENS, Université de Lyon, Lyon, France; ${ }^{4}$ Department of Medicine II, Division of Gastroenterology, University Medicine Rostock, Rostock, Germany; ${ }^{5}$ Department of Cardiac Surgery and Reference and Translation Center for Cardiac Stem Cell Therapy, University Medicine Rostock, Rostock, Germany; ${ }^{6}$ Department of Public Health and Community Medicine, Tufts University School of Medicine, Boston, Massachusetts; ${ }^{7}$ Perinatal Neuroepidemiology Unit, Hannover Medical School, Hannover, Germany. Correspondence: Olaf Wolkenhauer (olaf.wolkenhauer@uni-rostock.de)
} 
toward cell function and the underlying molecular mechanisms. The role of molecules in cell behavior and the role of cells within a tissue or an organ are inherently time-related phenomena. Therefore, new approaches were required because bioinformatics and statistical analyses are not sufficient to explore the time-dependent function of cellular systems. Dynamical systems theory has since provided the basis for kinetic and mechanistic modeling of processes at the subcellular, cell, tissue, and organ level (1). In the current review, we touch upon the computational aspects and clinical complexity of data gathering but focus primarily on mathematical modeling and (cell-) biological complexity underlying many disorders and diseases.

Although there are numerous examples of systems biology projects that address biomedical questions, it cannot be denied that most of the efforts are easily labeled as "basic research," often being pathway centered with a focus on the subcellular level $(2,3)$. Furthermore, we explore the challenges and limitations of mechanistic modeling in systems medicine in the following sections. Systems biology taught us about biological complexity with the practical consequence that in order to improve our understanding of biological (mal)function, one has to gather data from a wide range of technologies. There is no privileged perspective, level of biological organization, or associated technology. This need is carried over to systems medicine where there is then an obvious consequence of this development related to the "clinical complexity" of understanding disease: comorbidities-the presence of one or more disorders (or diseases) in addition to the primary disease or disorder-will require an even broader spectrum of data that need to be collected and analyzed.

Systems biology for medical applications does not represent the full deployment of systems medicine. Although biomedical or even clinically relevant research programs can be addressed by basic research, there is a recognized need to "translate" basic findings into clinical research. Instead of speaking about "translational research," we argue for the "integration of clinical and nonclinical data." The idea is not to translate ideas from one domain to another, such as translating advances in material science into improved consumer products, but to integrate data from in vitro experiments, animal models, and high-dimensional omics data with clinical data on body characteristics and function, biomarkers, and health history. This process is probably close to what Khoury et al. (4) refer to as "knowledge synthesis," the component of "knowledge integration" nestled between "knowledge management" (the decision-making process for which information to include) and "knowledge translation." The broad range and often heterogeneous data sets raise not only conceptual challenges; from a computational perspective, suitable interfaces are required to visualize and relate the data available, to allow or prepare a full analysis, selection, and interpretation. An important aspect, that increases the challenge even further, is the need to not only track biological parameters of patients before and after treatment but also to have available as much as possible of the patient's history. Although many decisions to date are based on single-point-in-time measurements, it is technically relatively easy to monitor patients and gather data over extended periods of time. Rather than deciding whether a patient has passed a statistically determined threshold (comparing the patient with the statistics of a population), a systems medicine approach ought to exploit the temporal dimension of trends in the development of biological parameters for individual patients. In pediatric research, the argument may be taken to the conclusion that the early development of a child determines the "initial conditions" for the emergence of disorders or diseases, and in order to allow a personalized diagnosis, prognosis, and therapy, one ought to know the trajectory that takes the patient into the disease state.

Examples of the clinical complexity, in which the tracking of a patient's molecular and physiological state(s) over an extended period of time is of central importance, are chronic diseases or disorders. Chronic diseases are a consequence of interactions between a body and genome with the environment, including lifestyle and other risk/protective factors, throughout a life. In the clinic, these diseases express themselves often through comorbidities, whereas at the molecular and cell levels, most probably identifiable specific mechanisms and their alterations are responsible for a loss of physiological function through the age of the body. To determine the measures of disease severity and control, genotypic and phenotypic patient-specific data need to be collected.

The outcome of studies in children and adults should therefore be prognostic markers that can be used to monitor "early warning signals" in patients during their lives. The integration of basic research and clinical practice is thus motivated by the need for an integration of clinical and nonclinical data. From a perspective of systems biology, an important lesson is that although many diseases have cellular and molecular bases, it is the combination of the cellular environment, the tissue in which the disease occurs, and the physiology of the organ or organs involved that largely determines pathogenesis and disease progression. In pediatric research, it also entails normal and abnormal developmental processes (5). Understanding such diseases requires not only knowledge of cells and subcellular processes but also a conceptual integration of data and models across multiple levels of structural and functional organization $(6,7)$. This in turn requires an integration of multiple kinds of expertise and data types from molecular and cell biology, physiology, clinical medicine, and epidemiology.

Depending on the kind of data (e.g., physiological measurements of body function vs. metabolomic spectra) and on the technologies used for data generation (e.g., deep sequencing vs. proteomics), a wide range of statistical and computational tools are required. Although technology-defined within each domain, data analysis methodologies are often well established. For their combination, however, new techniques are required to relate and interpret heterogeneous data sets. Furthermore, it will be essential to design computational platforms to manage, share, and integrate clinical and nonclinical data. To this end, ontologies and standardization play an increasingly important role for the management of data and models. In summary, systems medicine requires not only the integration of clinical and nonclinical data, of basic research and clinical knowledge, but also the integration of expertise from a wide range of disciplines. 


\section{Review | Wolkenhauer etal.}

We will discuss the notion of "integration" as a guiding principle for the development of a roadmap for systems medicine.

\section{DECIPHERING BIOLOGICAL COMPLEXITY AND MANAGING} CLINICAL COMPLEXITY REQUIRES INTERDISCIPLINARITY

Collaborations with bioinformaticians and statisticians have a long and successful track record in the medical sciences. Mathematical modeling, however, has still not achieved its full integration or even acceptance within the biomedical research community. An important reason for the success and acceptance of statistical and bioinformatical analyses is that they can often be conducted separately from the experiments. Following the identification and characterization of cellular components in the context of bioinformatics, the focus has shifted in recent years to the study of mechanisms that determine the function of cells in terms of gene regulatory networks, signal transduction, and metabolic pathways and networks. This shift of focus toward an understanding of functional activity and, therefore, toward cellular processes required methodologies from systems theory and, therefore, expertise from fields other than computer science and physics. The term "systems biology" has become associated with an interdisciplinary approach that reflects a practice of data-driven modeling and model-driven experimentation. With systems biology, mathematical models have become a central element in the formulation of biological arguments, and as a consequence, a new quality of interdisciplinary collaboration has become necessary. The "modeler" or "theoretician" no longer plays a simple supportive role. Instead, the construction and analyses of the models require both the "experimentalist" and the "modeler" to meet with an equal passion for a common interest and rely upon each other. The data are situated between the experimentalist and the modeler (8). To design experiments together and to interpret data together requires foremost computational platforms to manage, i.e., store, share, and visualize, the data. What is summarized by the term "data" here is in fact a vast array of data types, ranging from conventional diagnostics of blood and urine samples to patient-specific biological parameters, including hormones, endogenous viruses, molecular surface markers, and a range of genetic and functional genomic measurements. Although we wish to personalize diagnosis and therapy by tracking data for an individual to determine the patient's characteristic base levels and developmental trends, biomarkers will play an important role in stratifying patient groups to a level at which predictive markers can be used for individualized decision making. The long-term management of data and the protection of patient information from misuse raise enormous technical, conceptual, and data protection issues. Rather than exploring these aspects further, as important as they are, we shall here focus on one lesson from systems biology that is equally important for systems medicine to reach maturity but may easily be forgotten in light of the practical and technical challenges that we face.

New technologies have been a driving force behind advances in the life sciences. In practice, technologies are no longer considered simply a means to an end. Instead, the technologies often determine the questions that can be asked. Whether microarrays or recent developments in deep sequencing, it should not be forgotten that hypotheses about biomedical phenomena should come first, and only then, should one identify the means to test these hypotheses. Following a technology-driven phase, biomedical research has to face up to the fact that just as technologies are needed to generate data, novel methodologies are required to help us interpret the data. Data are not facts; facts are a matter for human interpretation in the context of prior knowledge. However, nonlinear dynamics and multilevel mechanisms are aspects of biological complexity that render common sense and intuition-as well developed as they might be-incapable of providing full understanding. The value of using the systems theory framework and established tools in systems medicine is that it is a way of thinking about the organization and emerging behaviors of complex systems.

Although nonlinear dynamics has been a focus of cell- and pathway-centric approaches in systems biology, the multilevel perspective is going to be the final frontier of systems medicine. High-impact advances in systems medicine will require an understanding of how tissue/organ dysfunction at the physiological level is related to cell functions at the molecular level. In the human body, properties of the organs and tissues emerge from the interactions of components at that level, and at lower and higher levels of spatial and temporal organization, both influenced by environmental changes. Therefore, it is likely that a better understanding of the emergence, progression, and treatment of a disease will need to involve novel approaches to mathematical modeling (9). To begin understanding structurefunction coupling across system levels instead of (or complementary to) ontological mechanistic modeling of biochemical and biophysical processes, we need a mathematical framework that helps us develop epistemological arguments about tissue organization. This requires a change in perspective that is less gene, pathway, and cell centered but instead puts the search for organizing principles back into the spotlight that guides our experiments.

Organizing principles are robust generalizations, similar to laws in physics that represent instantiations of biological general principles governing the evolution of the overall system (10). We can arrive at organizing principles through generalization-from in vitro to in vivo models, from a model organism to humans, from a particular cell type to cells in general, but also from inferences achieved with one technology to general observations. The search for organizing principles requires nonreductive strategies that provide explanations in terms of how categories of systems are organized in order to work. As a consequence of the underlying biological complexity, systems medicine must rely on the integration of evidence, data, and models across multiple levels of structural and functional organization. To this end, strategies are required to (i) develop and maintain computational platforms for the integration of clinical and nonclinical data; (ii) further develop technologies for quantitative and time-oriented tracking of changes in gene expression, cell signaling, and metabolism in relation to environmental influences; and (iii) develop methodologies for 
statistical analyses of integrated (spare and heterogeneous) data sets and mathematical concepts for models that integrate information across the multiple levels of structural and functional organization of a tissue, organ, or body.

The need to discuss integration in biology and biomedicine is becoming more urgent because the amount and variety of data required for understanding any given problem is steadily increasing. Moreover, many scientific questions require a range of expertise from different fields. Therefore, integration across disciplinary boundaries is crucial. The expertise for particular technologies and experimental systems is rarely found in a single laboratory, institute, or country, and this raises the need for standards and ontologies that support the sharing and integration of data and models.

\section{EN ROUTE TO SYSTEMS MEDICINE: INTEGRATION AS A GUIDING PRINCIPLE}

Integration is a combination of activities that provides a more comprehensive and coherent picture of complex research problems. These activities range from combining data, models, and methodologies to merging explanations and establishing closer connections among disciplines. A recent workshop on "Integration in Biology and Biomedicine" explored how integration works and how it is perceived by scientists and philosophers (11).

Most biomedical research projects are not capable of addressing complex problems directly and in a comprehensive way. Virtually all projects-even large-scale collaborative research efforts-are forced to "zoom in" on a specific aspect, subsystem, or narrowly defined question. Taking cancer research as an example, resistance to cell death is one aspect of understanding carcinogenesis and tumor progression. Although a very large number of research projects would define their goals in these terms, in practice, virtually all projects in molecular and cell biology take a reductionist strategy by focusing on a particular molecule or a particular (usually single) pathway. Often, they use a specific technology in the context of a particular experimental system, thereby creating a rather narrow context in which data can be interpreted.

Biological complexity has forced molecular and cell biology into reduction and contextualization, with an ever-increasing degree of specialization. What is frequently lost or forgotten along this road of reduction, contextualization, and specialization is the original question that led to such highly focused research projects. Strategies and concepts are required that can guide the integration of evidence and results into the formulation of theories, hypotheses, and explanatory models (12). At present, this process of integration of evidence into the formulation of organizing principles (robust generalizations) is left to a few talented authors of leading-edge review articles, but these are few and far between. The importance of review articles in the life sciences is evidence for the need to develop strategies that can guide the search for organizing principles that are underlying the (mal)functioning of cellular systems.

Integration of data has always been important in biology and biomedicine, but in most cases, it is performed on an ad hoc basis, often with a lack of recognition of the importance of the insights that integration makes possible. The inclusion of philosophy in the design of roadmaps for systems medicine could facilitate crucial reflection on scientific practice and thereby aid conceptual development and integrative efforts in interdisciplinary collaborations. The philosopher's awareness for language and argument can also play an active role in the development of ontologies, which are central for the development of standards that are required to share and integrate data from heterogeneous sources and across disciplinary boundaries.

The "Toolbox Project" of Michael O'Rourke and Stephen Crowley (http://www.cals.uidaho.edu/toolbox/) is an example for the integration of expertise in cross-disciplinary challenges. Rooted in philosophical analysis, the Toolbox Project enables a structured, questionnaire-based dialogue in which participants explore epistemic and conceptual differences of their fields and working practice. This yields both awareness for the language and culture of their specializations and mutual understanding as a basis for effective collaborative research.

Integration requires a strategic effort from the scientific community and the funders of science. Without mechanisms to encourage truly interdisciplinary large-scale collaborative efforts, the forms of integration discussed here will not become reality. A common misconception about modeling is that it can save time and money and render experimental work unnecessary. It is true that a mechanistic model allows simulation experiments in which initial conditions, the values of model parameters, and even the structure of the model can be varied to explore the behavior of the system under consideration. What is often forgotten is the experimental effort required to establish the model in the first place. For dynamical systems, the experimental effort to generate sufficiently rich, quantitative time course data will usually be far higher than the average experiments of the past.

Interdisciplinary projects are likely to take more time than a project in a single discipline. This will have consequences for the design of $\mathrm{PhD}$ programs and the evaluation of career performance of scientists. The interdependence among project partners makes it necessary that teams are not just a match of expertise but a match of minds and hearts dedicated to jointly tackling important and difficult biological and medical questions. The sociological element of such large-scale efforts, which are doubtlessly necessary for medicine to advance, brings with it a high risk of failure. Although for systems biology, many projects have been characterized by collaborations with typically two partners-an experimentalist and a modeler-in systems medicine, teams are expected to cover a broader range of expertise. A typical project that aims at integrating data from basic research with patient background data and clinical information is likely to require a team involving a clinician, a pathologist, a molecular biologist, a computer scientist, an epidemiologist, a statistician, and a mathematician. Needless to say, this raises a number of practical challenges, and if these problems are not addressed by research-funding bodies, such integrative projects are unlikely to occur in the first place and less likely to succeed in the long run. For an efficient and successful implementation of systems medicine 
programs, it will thus be valuable to reflect upon epistemological, ontological, and sociological aspects of highly interdisciplinary endeavors.

\section{ROADMAP FOR SYSTEMS MEDICINE}

Personalized medicine is frequently considered one of the primary goals for systems medicine, and it has been argued that such a medicine-also required to be predictive, preventative, and participatory-cannot be achieved by an incremental evolution but requires a revolutionary change in the practice of medicine (13). Central to these objectives are systems approaches that provide clinicians with access to an integrated high-dimensional personal data set of a patient and to enable clinicians to link this personal data set to information from research databases. In essence, if diseases are conceptualized as network perturbations, then patient data need to be looked at using the tools of network analysis (14). This vision comes along with enormous technical, methodological, and legal challenges. We therefore focus on only one particular methodological challenge related to the conceptual integration of data and the role mathematical modeling can play in dealing with biological complexity.

In recent years, much has been learned about molecular components and subcellular processes. Still, one important question that remains to be answered is how can we integrate data and models across a wide range of spatial and temporal scales? Although systems biology is an approach by which biological questions are addressed through integrating experiments in iterative cycles with computational modeling, simulation, and theory (15), systems medicine carries this approach forward into a disease-oriented era, driven by clinical and public health needs (16).

Mathematical modeling enables the integration of biological and clinical data at various levels. The value of modeling lies in the fact that it necessitates the statement of explicit hypotheses, a process that often improves our understanding of the system under consideration and uncovers critical points of poor understanding. Furthermore, simulations of models can reveal hidden patterns and/or counterintuitive mechanisms in complex systems. Theoretical thinking and mathematical modeling can thus help generate new hypotheses that can be tested in the laboratory. Experience has shown that success in systems biology was achieved when quantitative data became available, and even simple mathematical models have been shown to be of practical use. It is very important to recognize that the interdisciplinary process leading to the formulation of a model has intrinsic value in and of itself (17).

However, before mathematical modeling can be used, data need to be made available in quantitative and standardized form and be subjected to statistical analyses in order to quantify data variability. The data that emerge from these preprocessing steps provide the basis for mathematical models. The art of modeling lies not in the accuracy of the model but in the explanation the model provides and the subsequent argument it enables researchers to formulate. This biological argument to which a model contributes, in turn, lends credibility and validity to the model. As opposed to the use of models in the engineering sciences, we believe that in systems medicine, the main purpose of models will not be numerical prediction but enhancement of our understanding by managing uncertainty arising from biological complexity, reflected in heterogeneous data, nonlinear dynamics, and the multilevel nature of biological processes.

Data reflecting the patient's health are as important to therapeutic decisions as they are for the development of drugs. A successful systems medicine requires advances in understanding the functioning of drugs-their therapeutic value and their toxicity. This in turn requires a better understanding of cellular- and tissue-level networks, linked to an individual patient's genome, metabolome, and proteome. It will be necessary to depart from gene-, protein-, and pathway-centric approaches (18).

Genomics has played an important role in the life sciences and will continue to play a role in systems medicine. Genomics itself, however, does not provide a means to unravel the complex mechanisms underlying cellular networks, nor will genomics data be sufficient to understand the action of drugs, on the basis of which we can develop monotherapy or combination therapy, or alter the pathophysiology of disease so as to maximize therapeutic benefit and minimize toxicity. To this end, an integrative approach is necessary, bringing together investigators from computational biology, systems biology, and biomedical engineering. Mathematical modeling is only one pillar that is required to support systems medicine. Statistical analyses and bioinformatics tools are equally important and are more widely accepted and used in medicine. Systems medicine provides a new challenge to interdisciplinary research, something that needs the attention of research-funding bodies.

Although roadmaps are being devised for new funding programs in systems medicine, we are well advised to remember that roads emerge as we move along the path toward integrative approaches. In other words, it is impossible to define more than the starting points and goals for systems medicine (as we have done above). The roads will necessarily remain unclear until we allow scientific creativity to take its course. Nevertheless, it should be possible to describe a few milestones that can be expected to serve as signals that progress has been made. Suggested "milestones" (not in order of expected achievement) are:

1. Consensus on what defines solid evidence in disease causation research (etiopathogenetic dimension) and intervention effectiveness research (therapeutic dimension).

2. Consensus on what kinds of data need to be integrated to achieve a level of evidence that can be accepted by the diverse research communities.

3. Consensus on whether and how intrapersonal (single patient) and interpersonal (epidemiologic) data can be integrated.

4. Consensus on how best to perform such large-scale, interdisciplinary research might require new kinds of research institutions integrated in collaborative networks. 


\section{ACKNOWLEDGMENT}

Parviz Minoo served as the deciding editor for this article.

\section{STATEMENT OF FINANCIAL SUPPORT}

O.W. acknowledges support from the German Research Foundation (DFG), grant WO 991/10-1, and the German Federal Ministry for Education and Research (BMBF) as part of their systems biology programs. C.A. acknowledges support by the CNRS and the EU grants to the MeDALL (Mechanisms of the Development of Allergy) consortium (grant FP7 №264357), and the U-BIOPRED (Unbiased Biomarkers for the PREDiction of respiratory disease outcomes) consortium (grant IMI 115010). The European Institute for Systems Biology and Medicine hosted at Claude Bernard University is supported by the Lyonbiopole competitive cluster and its academic, industrial, and local authority partners, including Grand Lyon, Région Rhône-Alpes, Direction de la Recherche et de la Technologie, and the Finovi Foundation. C.A. and O.W. are partners of the EU-FP7 Coordination Action CASyM (Systems Medicine Implementation of Systems Medicine across Europe) (grant 305033). During the writing of this article, O.D. was supported by a cooperative agreement with the National Institute of Neurological Disorders and Stroke (ELGAN, NS040069) and by the European Union (NEUROBID, grant 241778; NEO-CIRC, grant 282533).

\section{REFERENCES}

1. Wolkenhauer O, Fell D, De Meyts P, et al. SysBioMed report: advancing systems biology for medical applications. IET Syst Biol 2009;3:131-6.

2. Wolkenhauer O, Auffray C, Baltrusch S, et al. Systems biologists seek fuller integration of systems biology approaches in new cancer research programs. Cancer Res 2010;70:12-3.

3. Kreeger PK, Lauffenburger DA. Cancer systems biology: a network modeling perspective. Carcinogenesis 2010;31:2-8.

4. Khoury MJ, Gwinn M, Dotson WD, Schully SD. Knowledge integration at the center of genomic medicine. Genet Med 2012;14:643-7.

5. Dammann O, Follett P. Toward multi-scale computational modeling in developmental disability research. Neuropediatrics 2011;42:90-6.

6. Tegnér JN, Compte A, Auffray C, et al. Computational disease modeling fact or fiction? BMC Syst Biol 2009;3:56.
7. Kohl P, Noble D. Systems biology and the virtual physiological human. Mol Syst Biol 2009;5:292.

8. Wolkenhauer O, Hofmeyr J. Interdisciplinarity as both necessity and hurdle for progress in the life sciences. In: Andersen H, Dieks D, Gonzales WJ, Uebel T, Wheeler G, eds. New Challenges to Philosophy of Science - The Philosophy of Science in a European perspective 4. Amsterdam, The Netherlands: Springer Verlag, in press.

9. Cohen JE. Mathematics is biology's next microscope, only better; biology is mathematics' next physics, only better. PLoS Biol 2004;2:e439.

10. Wolkenhauer O, Shibata D, Mesarovic MD. The role of theorem proving in systems biology. J Theor Biol 2012;300:57-61.

11. Green S, Wolkenhauer O. Integration in action. EMBO Rep 2012;13: 769-71.

12. Dammann O, Gressens P. Integrated mechanism reviews. Pediatr Res 2012;71:530-1.

13. Hood L, Balling R, Auffray C. Revolutionizing medicine in the 21st century through systems approaches. Biotechnol J 2012;7:992-1001.

14. del Sol A, Balling R, Hood L, Galas D. Diseases as network perturbations. Curr Opin Biotechnol 2010;21:566-71.

15. ERASysBio Partners. Systems biology in the European research area (a report - March 2008). (http://www.erasysbio.net/lw_resource/datapool/_ pages/pdp_2/ERASysBio_Systems_Biology_Strategy_Paper_25-Mar2008.pdf). Accessed 1 October 2012.

16. Auffray C, Chen Z, Hood L. Systems medicine: the future of medical genomics and healthcare. Genome Med 2009;1:2.

17. European Science Foundation. Advancing systems biology for medical applications (a science policy briefing no 35 - December 2008). (http:// www.esf.org/nc/research-areas/medical-sciences/activities/sciencepolicy/advancing-systems-biology-for-medical-applications.html). Accessed 1 October 2012.

18. National Institute of General Medical Sciences. Quantitative and systems pharmacology in the post-genomic era: new approaches to discovering drugs and understanding therapeutic mechanisms (an NIH white paper by the QSP workshop group - October 2011). (http://www.nigms.nih.gov/ NR/rdonlyres/8ECB1F7C-BE3B-431F-89E6-A43411811AB1/0/SystemsPharmaWPSorger2011.pdf). Accessed 1 October 2012. 